\title{
Variation in Osteoid Pattern With Age in Human Vertebrae
}

\author{
By \\ Jung-Kuei Wei and James S. Arnold \\ Department of Anatomy, School of Medicine, Keio University, Tokyo, Japan \\ and Department of Pathology, Kansas City General Hospital, \\ Kansas City, Missouri, U. S. A. \\ -Received for Publication, February 29, 1972-
}

The loss of trabecular bone from the vertebra has long been recognized as an early feature of osteoporosis (Albright et al., '41). Bone loss is a result of a greater rate of resorption than replacement by new bone. In spite of the obvious clinical importance of trabecular bone remodeling and consequent morphological change of the internal structure of the vertebrae with age, only few studies have been reported dealing with this problem (Arnold ot al., '68, Atkinson, '67, Casuccio, '72a).

In the progress of osteoporosis some trabeculae are thinned and destroyed. Others undergo thickening and are converted to flat platelike structures. This process has been referred to as compensatory hypertrophy or hypertrophic atrophy (Casuccio, '62b, Steinbach, '64). In the authors' previous investigation (Arnold et al., '68) the aging change of the various trabecular structures of the vertebra was described in detail. With their new knowledge of different types of trabecular structures and their different rate of loss with age, the authors wished to determine which of the structures was turning over most rapidly as a function of age. In order to accomplish this goal, a new method of osteoid staining which makes it possible to demonstrate topographically the distribution of bone-forming sites in a vertebra was developed (Wei and Arnold, '70).

The present investigation was done to describe the changing pattern of bone formation at the surfaces of various types of trabeculae and their location within the vertebral bodies, as a function of age, using the above-mentioned technique of osteoid staining. 


\section{Materials and Methods}

Specimens of human lumbar vertebrae were obtained at autopsy. Thirty males and 19 females ranging from 8 months to 98 years of age were examined. All had died within three days of acute illness or trauma such as acute hemorrhage, burn, suicide or car accident (table 1). Subjects with chronic renal, endocrine, hepatic or heart disease, which might be considered to have some effect on bone, were excluded.

Table 1. Age distribution and case of death in studied cases.

\begin{tabular}{|c|c|c|c|c|c|c|c|c|c|c|}
\hline \multirow{2}{*}{ Age } & \multicolumn{3}{|c|}{ Sex } & \multirow{2}{*}{ Suicide } & \multirow{2}{*}{$\begin{array}{l}\text { Trauma } \\
\text { (Accident) }\end{array}$} & \multirow{2}{*}{$\begin{array}{l}\text { Intra- } \\
\text { Cerebral } \\
\text { Hemor- } \\
\text { rhage }\end{array}$} & \multirow{2}{*}{$\begin{array}{c}\text { Poison } \\
\text { ing }\end{array}$} & \multirow{2}{*}{$\begin{array}{c}\text { Acute } \\
\text { Pneumo } \\
\text { nia }\end{array}$} & \multirow{2}{*}{$\begin{array}{c}\text { Acute } \\
\text { Bleed- } \\
\text { ing }\end{array}$} & \multirow{2}{*}{ Others* } \\
\hline & $\mathbf{M}$ & $\mathrm{F}$ & Total & & & & & & & \\
\hline $0-10$ & 6 & 2 & 8 & & 3 & & 1 & 3 & & 1 \\
\hline $11-20$ & 5 & 0 & 5 & 1 & 3 & & & & & 1 \\
\hline $21-30$ & 6 & 1 & 7 & 1 & 3 & 1 & & 1 & 1 & 1 \\
\hline $31-40$ & 4 & 0 & 4 & & 1 & 1 & 1 & & & 1 \\
\hline $41-50$ & 1 & 5 & 6 & 2 & 1 & 1 & 1 & & & 1 \\
\hline $51-60$ & 2 & 2 & 4 & & 1 & 3 & & & & \\
\hline $61-70$ & 2 & 3 & 5 & & 1 & 4 & & & & \\
\hline $71-80$ & 4 & 2 & 6 & 1 & 2 & & & 1 & 1 & 1 \\
\hline $81-90$ & 0 & 3 & 3 & 1 & & 1 & & 1 & & \\
\hline 91- & 0 & 1 & 1 & & 1 & & & & & \\
\hline Total & 30 & 19 & 49 & 6 & 16 & 11 & 3 & 5 & 2 & 6 \\
\hline
\end{tabular}

$\begin{array}{lll}* \text { Others : } & \text { Cerebral occlusion } & 2 \\ & \text { Unexplained acute death } & 4\end{array}$

Approximately $7 \mathrm{~mm}$ thick, fresh midsagittal slabs were cut from first lumber vertebrae as shown in fig. 1. Cross sections of the second vertebrae were also made in some cases. They were prepared and stained by technique previously described (Wei and Arnold, '70). A brief description of the procedures used is as follows:

After washing out bone marrow and soft tissue in a jet stream of water, the slab was immersed in $0.5 \%$ basic fuchsin in $30 \%$ alcohol solution for $30-40$ hours at $20^{\circ} \mathrm{C}$. In order to remove overstained surfaces and make a suitable thickness for inspection, the slab was trimmed by cutting off both surfaces with a diamond saw, leaving a $2 \mathrm{~mm}$ thick slab from the middle portion. The osteoid was distinguished from the diffuse surface-staining by differentiating in $50 \%$ ethyl alcohol until the nonosteoid trabecular surfaces turned light pink while the osteoid remained dark red. The specimens were then dried 
in air and kept in a dark place without mounting. Inspection was performed under a dissecting microscope with the specimen immersed in water buffered at $\mathrm{pH} 7.0-8.0$. For the quantitative study of the extent of surface covered with osteoid, a random point-counting method was used (Chalkley, '43). The specimen was viewed under the dissecting microscope containing an eyepiece with rectangular grids using diffus $\theta$ incident illumination (fig. 2). The grids were moved progressively over the specimen to give a total of 50 fields without virtual overlap. The number of hits (line-intersections) falling on osteoid-covered and nonosteoid trabecular surfaces were separately recorded. This procedure was then repeated on the opposite side of the specimen and the two readings were averaged. The percentage of osteoid was calculated as follows:

$\frac{\text { Hits over osteoid-covered surfaces }}{\text { Hits over osteoid-covered plus nonosteoid surfaces }} \times 100$.

\section{Observations}

1) Childhood (1st and 2nd decades)

In one infant and in younger children, the vertebra is square in form and shows smooth corners. Trabeculae are regularly arranged as in young adults, except for the transverse trabeculae, which have a tendency to form arch-like arrangements around the center of the vertebra instead of running straight across the vertebral body. Some plate-like trabeculae are found around the central vascular space. Osteoid is abundant and distributed all over the vertebra, regardless of the type or location of the trabeculae. Plate-like trabeculae around the central vein (basi-vertebral vein) are also covered with osteoid. In cross sections, the osteoid is often found as a ring circumscribing the round bone marrow cavity. The average percentages of trabecular surface occupied by osteoid are $29.02 \%$ in the 1 st decade and $14.86 \%$ in the 2nd decade (table 2).

2) Young adult (3rd and 4th decades)

In young adults, the upper and lower horizontal thirds (end zones) of the sagittal section are composed of regularly arranged trabeculae forming rectangular grids separating the bone marrow into small squares (fig. 3). Immediately beneath the end plates, these squares appear small but become larger toward the central zone of the vertebra. Plate-like structures are found surrounding the basivertebral vein, but in the mid-anterior portion of the vertebra they form a fuzzy triangle. The studied vertebrae are mostly rectangular in shape and show no deformities such as spurring or cupping. 
Little osteoid is observed in this period of life. The osteoid present is usually seen on the surfaces of transverse trabeculae (especially slender ones) rather than on longitudinal trabeculae (fig. 5). In addition, osteoid has a tendency to concentrate on the trabeculae located in the deeper portion at the center of the vertebral body. The turnover rate of bone must be very slow in this age group, because bone formation is scarce, and loss of bone has not yet begun. The large plate-like structures usually lack osteoid, although in some cases small patches of osteoid are found sporadically on the thinnest parts and edges. The average percentage of trabecular surfaces with osteoid is found to be $6.74 \%$ in the twenties (average of 7 cases) and $8.72 \%$ in the thirties (average of 4 cases) (table 2).

Table 2. Percentage of Osteoid.

\begin{tabular}{|c|c|c|c|c|c|c|c|c|c|c|c|}
\hline $\begin{array}{l}\text { Case } \\
\text { No. }\end{array}$ & Age & Sex & $\begin{array}{c}\text { Osteoid } \\
(\%)\end{array}$ & $\begin{array}{l}\text { Case } \\
\text { No. }\end{array}$ & Age & Sex & $\begin{array}{c}\text { Osteoid } \\
(\%)\end{array}$ & $\begin{array}{l}\text { Case } \\
\text { No. }\end{array}$ & Age & Sex & $\begin{array}{c}\text { Osteoid } \\
(\%)\end{array}$ \\
\hline 1 & $4 \mathrm{mo}$. & $\mathbf{M}$ & 47.2 & 18 & 28 & F & 8.6 & 35 & 64 & $\mathrm{~F}$ & 26.4 \\
\hline 2 & 1 yr. & $\mathbf{M}$ & 35.4 & 19 & 29 & $\mathbf{M}$ & 6.4 & 36 & 65 & $\mathbf{M}$ & 13.5 \\
\hline 3 & 2 & $\mathbf{M}$ & 24.5 & 20 & 29 & M & 4. 2 & 37 & 66 & F & 8.9 \\
\hline 4 & 3 & $\mathbf{M}$ & 32.8 & 21 & 31 & M & 9.8 & 38 & 69 & $\mathbf{M}$ & 31.3 \\
\hline 5 & 7 & $\mathrm{~F}$ & 18.9 & 22 & 31 & M & 3.2 & 39 & 70 & F & 19.6 \\
\hline 6 & 7 & $\mathrm{~F}$ & 28.4 & 23 & 32 & $\mathbf{M}$ & 12.6 & 40 & 71 & M & 28. 1 \\
\hline 7 & 8 & $\mathbf{M}$ & 23.8 & 24 & 39 & $\mathbf{M}$ & 7.5 & 41 & 71 & $F$ & 13.3 \\
\hline 8 & 10 & $\mathbf{M}$ & 21.2 & 25 & 42 & $\mathrm{~F}$ & 17.1 & 42 & 72 & $\mathrm{~F}$ & 14.0 \\
\hline 9 & 12 & $\mathbf{M}$ & 30.3 & 26 & 42 & $F$ & 6.5 & 43 & 72 & $\mathbf{M}$ & 8.6 \\
\hline 10 & 14 & $\mathbf{M}$ & 13.8 & 27 & 43 & $F$ & 9.8 & 44 & 75 & $\mathbf{M}$ & 30.2 \\
\hline 11 & 17 & $\mathbf{M}$ & 14.8 & 28 & 47 & $\mathbf{M}$ & 16.7 & 45 & 79 & $\mathbf{M}$ & 17.1 \\
\hline 12 & 17 & $\mathbf{M}$ & 8.8 & 29 & 47 & $\mathrm{~F}$ & 7.2 & 46 & 81 & $F$ & 10.6 \\
\hline 13 & 19 & $\mathbf{M}$ & 6.6 & 30 & 49 & $\mathrm{~F}$ & 12. 3 & 47 & 81 & $\mathrm{~F}$ & 20.2 \\
\hline 14 & 24 & $\mathbf{M}$ & 7.8 & 31 & 52 & $\mathbf{M}$ & 16.1 & 48 & 88 & $\mathrm{~F}$ & 18.4 \\
\hline 15 & 25 & $\mathbf{M}$ & 5.4 & 32 & 53 & $\mathrm{~F}$ & 28.5 & 49 & 98 & $F$ & 15.0 \\
\hline 16 & 26 & $\mathbf{M}$ & 12.3 & 33 & 54 & F & 10.3 & & & & \\
\hline 17 & 27 & $\mathrm{M}$ & 2. 6 & 34 & 60 & $\mathbf{M}$ & 19. 6 & & & & \\
\hline
\end{tabular}

3) Middle age (5th and 6th decades)

During this period of life the transverse trabeculae are seen to become thinner and some coarse, thickened longitudinal trabeculae are found in the center and posterior portion of the central zone. Osteoid is mostly seen on the slender transverse trabeculae. Some thickened longitudinal trabeculae are also partly covered with osteoid (fig. 6). In general, the vertebra which has more slender transverse trabeculae shows the higher percentage of osteoid since these thinner disappearing transverse trabeculae are the structures where osteoid is chiefly 
located. In some cases where cupping or spurring are present, osteoid is seen to be increased in amount on the trabeculae immediately beneath the end plate. This suggests that when such lesions develop, a prolonged period of remodeling is initiated in adjacent bone, producing structural changes necessary to withstand the stress and strain as well as to repair the damage caused by injury. The average percentage of trabecular surfaces with osteoid is $11.6 \%$ in the 5 th decade and $18.3 \%$ in the 6 th decade.

4) 7th and 8th decades

During this period of life, the aging change of trabecular pattern becomes obvious and can clearly be observed, although there is a variation with regard to the degree of atrophic change from case to case. In general, marked narrowing and loss of trabeculae are distinctive. The pattern and abundance of osteoid vary with the change of trabecular structures. More osteoid is found at the surface of the longitudinal trabeculae than in those of young adults. The coarse, thickened longitudinal trabeculae and plates are frequently covered with osteoid. In addition, the transverse and oblique trabeculae are also frequent sites of osteoid coverage. The corners of the vertebra where spurring has occurred are often seen to have thickening trabeculae covered by large quantities of osteoid. The average percentage of osteoid on the trabeculae surfaces is $19.9 \%$ in the 7 th decade, $18.7 \%$ in the 8 th decade.

5) 9th and 10th decades

Aging changes are conspicuous and show a marked variation in extent from case to case. Both atrophy and compensatory hypertrophy can be seen clearly in these decades. The remaining longitudinal trabeculae are mostly converted to plates and are frequently covered with osteoid. However, the basic pattern of osteoid is the same as in the 7 th and 8th decades. The average percentage of osteoid on the trabecular surfaces in these decades is $16.0 \%$.

\section{Discussion}

The fact that the existence of osteoid reflects the presence of osteoblastic activity and new bone formation has been checked by many investigators using tetracycline-labelled materials (Frost and Villanueva, '60 ; Kelly et al., '66 ; Manson and Lucas, '66 ; Milch et al., '57; Villanueva et al., '68). They proved that tetracyline-labelled new bone always corresponds to the presence of osteoid and no tetracycline-labelled new bone exists without accompanying osteoid 
in the material. Therefore, the presence of osteoid is a good index of the extent to which new bone formation is occurring.

In the authors' observation, the percentage of trabecular surfaces covered with osteoid in the vertebra is highest in infancy and then slows down in early adulthood. After that, it remains at its lowest value until age 40, as shown in fig. 4. This finding agrees with the description of Villanueva et al. ('63) in cortical bone of the rib. The explanation for the abundance of osteoid found in infancy and childhood is that the bone formation rate is highest in those ages and appositional growth of bone predominates before skeletal maturity is reached (Casuccio, '62a). Growth remodeling virtually ceases at around age 20 when bone formation and resorption are in equilibrium. Therefore, osteoblastic activity is low until involutional changes begin at age 40 (Bromley et al., '66). The increase in percentage of osteoid in the middle age was reported by Frost and Villanueva ('61) and Villanueva et al. ('63) in cortical bone of the rib. They demonstrated that the number of osteons covered with osteoid in a unit area of a cross section reaches the maximum in adult life at age 65. This result does not support the generally held anabolic hormone theory (Albright, '47). Frost and Villanueva ('61) inferred that such an increase of osteoblastic activity is in part the result of diminished action of adrenal cortical and thyroid hormones on bone remodeling. As Frost ('63) has pointed out, an increase in bone formation in cortical bone is always preceded by a resorption event (the formation of a resorption cavity). The authors believe that resorption activity virtually precedes bone formation in trabecular bone remodeling just as in cortical bone. Thus, an increase in bone formation must reflect an increased bone resorption. Similarly, it must be concluded that more bone surfaces are being resorbed with aging since more trabeculae are involved in bone formation. Of course, after 40 years of age there is a progressive loss in bone mass with aging (Caldwell, '62). Since the rate of change in bone mass is the difference between the rates of formation and resorption, the conclusion must be that the rate of bone resorption exceeds that of formation. In the present study the authors are measuring not the rate of bone formation but the fraction of trabecular surfaces which are engaged in the deposition of new bone. The fraction of the surfaces engaged in bone formation does not, per se, indicate how much new bone is being deposited. Fortunately, the authors' dilemma was resolved when Frost ('63) demonstrated that the rate of bone apposition in microns per day in man remains virtually constant throughout adult life. Thus, it can be safely assumed that increased osteoid at trabecular surfaces in the middle and old aged does in 
fact indicate a real increase in the rate of bone deposition (grams per day) and not simply an increase in the rate of apposition (microns per square centimeter per day).

The second contribution of the present study is the demonstration that bone formation does not occur randomly at trabecular surfaces as has been generally concluded from histologic studies using thin sections of bone. The authors three dimensional macroscopic observations of osteoid at trabecular surfaces have shown that bone formation is taking place at definite selected structures at some ages and at other structures at other ages. This finding suggests that local factors such as mechanical stress are of the greatest importance in determining where remodeling of bone occurs. This does not in any way deny the importance or existence of systemic regulatory factors, such as hormones. It does suggest, however, that systemic factors act by modifying the extent of the response to these mechanical stress factors.

It was also found that trabecular structures which are being lost from bone are often covered with osteoid, indicating that they have a high rate of bone formation at their surfaces. Since an increase in bone formation must reflect an increase in bone resorption, the explanation for the increased osteoid at the surfaces of transverse trabeculae after age 40 is that an amount of resorption activity must be taking place at the surfaces of these trabeculae.

\section{Summary}

The change in extent and pattern of new bone formation with age was studied by quantitative and qualitative observation of osteoid covering vertebral trabecular surfaces. The various types of trabecular structures, such as transverse and longitudinal trabeculae, were separately studied by a technique of staining thin slabs of vertebrae. The percentage of osteoid is highest in infancy and then decreases to a minimal value in early adulthood. It remains at this basal level through age 40 . During the 5th through 7th decades, formation increases three-fold above the basal level as progressive loss of bone mass occurs. In infancy and childhood, osteoid seams are evenly distributed throughout the vertebra, regardless of the type or location of the trabecular structures. However, in young adults, osteoid is small in amount and is seen only at the surfaces of the transverse trabeculae. In the 5th and 6th decades, osteoid is chiefly on the transverse trabeculae, indicating the high turnover of these structures which are being lost first in the involutional process. With decreasing mass of bone in aging, persisting longitudinal 
trabeculae are converted into plates by apposition of new bone, which is reflected by the presence of osteoid surfaces on longitudinal trabeculae in old age.

\section{Literature cited}

Albright, F., Smith, P. H. and Richardson, A. M. 1941. Postmenopausal osteoporosis : Its clinical features. JAMA, $11: 2465-2474$.

Albright, F. 1947. The effects of hormone on osteogenesis in man. Recent Progr. Hormone Res., $1: 293$.

Arnold, J. S., Bartley, M. H., Tont, S. A. and Jenkins, D. P. 1966. Skeletal changes in aging and disease. Clin. Orthop., 49: 17-38.

Arnold, J.S., Wei, J. K., Cochran, T. H., McNeill, G. C., Ament, C. M. and Gitta, P.S. 1968. External and trabecular morphologic changes of lumbar vertebrae in aging. NIH Symposium, "Progress in Methods of Bone Mineral Measurement".

Atkinson, P. J. 1967. Variation in trabecular structure of vertebrae with age. Calc. Tiss. Res., $1: 24-32$.

Bromley, R. G., Dockum, N. L., Arnold, J. S. and Jee, W. S. S. 1966. Quantitative histological study of human lumbar vertebrae. J. Gerot., $21: 537-543$.

Caldwell, R. A. 1962. Observations on the incidence, etiology and pathology of osteoporosis. J. Clin. Path., 15 : 421-431.

Casuccio, C. 1962a. An introduction to the study of osteoporosis (Biochemical and Biophysical research in bone aging). Proc. Poyal Soc. of Med. 55: 663-668.

Casuccic, C. 1962b. Concerning osteoporosis. J. Bone and Joint Surg., 44B : 453.

Chalkley, H. W. 1943. Method for the quantitative morphologic analysis of tissue. J. Nat. Canc. Inst., $4: 47-53$.

Frost, H. M., Villanueva, A. R. and Roth, H., 1960. Tetracycline staining of newly forming bone and mineralizing cartilage in vivo. Stain Tech., 35: 135-138.

Frost, H. M. and Villanueva, A. R. 1961. Human osteoblastic activity I. Henry Ford Med. Bull., $9: 76-86$.

Frost, H. M. 1963. Bone Remodeling dynamics. Charles C. Thomas, Springfield.

Kelly, P. J., Jowsey, J. and Riggs, B. L. 1966. A comparison of different morphologic methods of determining bone formation. Clin. Orthop., $40: 7-11$.

Manson, J.D. and Lucas, R.B. 1966. A microradiographic study of age changes in the human mandibula. Arch. Oral Biol., $7: 761-769$.

Milch, R. A., Rall, D. P. and Tobie, J.P. 1957. Bone localization of tetracycline. J. Nat. Canc. Inst., $19: 87$.

Steinbach, H. L. 1964. The roentgen appearance of osteoporosis. Radiol. Clin. N. Amer., 2 : 191-207.

Villanueva, A. R., Frost, H. M., Ilricki, L., Frame, B., Smith, R. and Arnstein, R. 1966. Cortical bone dynamics measured by means of tetracyćline labeling in 21 cases of osteoporosis. J. Lab. Clin. Med., 68 : 599-616.

Villanueva, A. R., Sedlin, E. and Frost, H. M. 1963. Variation in osteoblastic activity with age by the osteoid seam index. Anat. Rec., $146: 209-213$.

Wei, J.K. and Arnold, J.S. 1970. Staining osteoid seams in thin slabs of undecalcified trabecular bone. Stain Tech., $45:$ 193-198. 


\section{PLATE}




\section{Explanation of Figures}

Fig. 1. Dotted lines indicate the slab to be cut from a vertebral body.

Fig. 2. Point-counting method. The black-and-white arrow points at a hit (a lineintersection falling on a trabecula covered with osteoid).

Fig. 3. The mid-sagittal slab of the first lumbar vertebral body from a 25-year-old : man.

Fig. 4. Percentages of bone surface occupied by osteoid.

Fig. 5. Osteoid (dark red) is mostly seen on transverse trabeculae (34-year-old: woman).

Fig. 6. Some thickened longitudinal trabeculae are also covered with osteoid. (51-year-old man). 


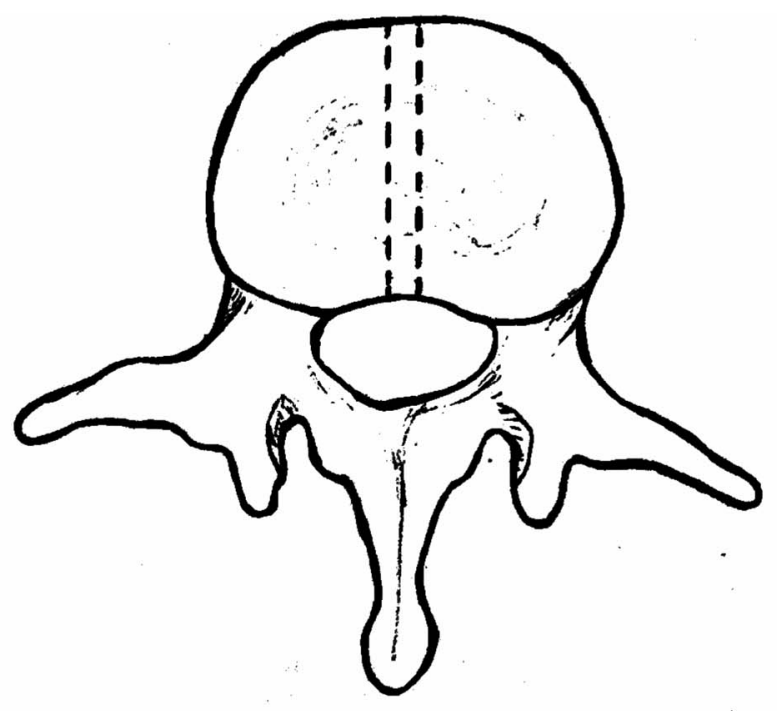

Fig.1.

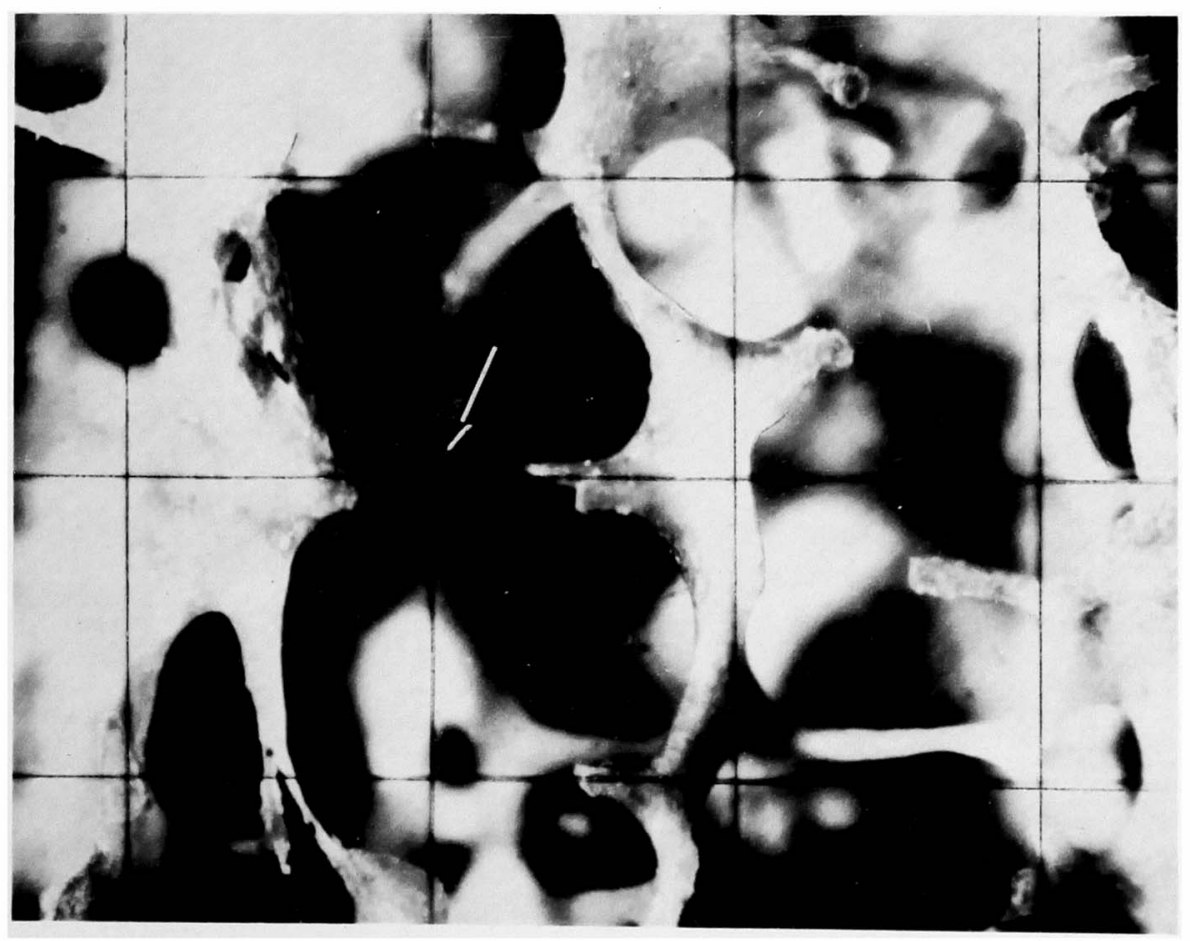

Fig.2.

\section{J. -K. Wei and J. S. Arnold}




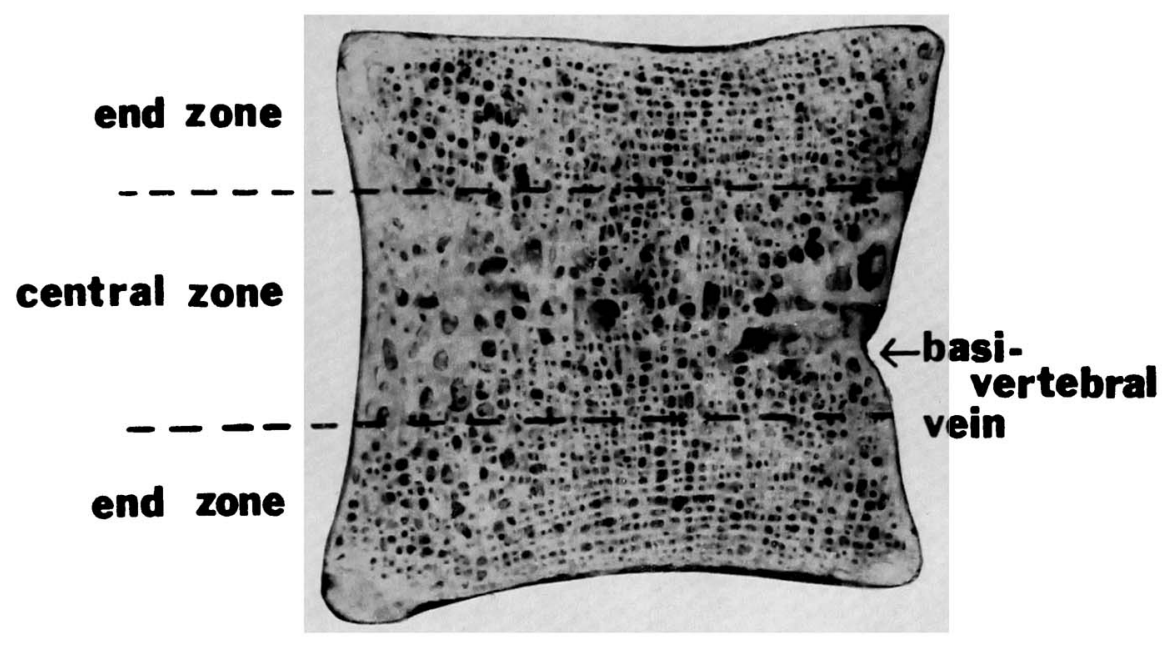

Fig.3.
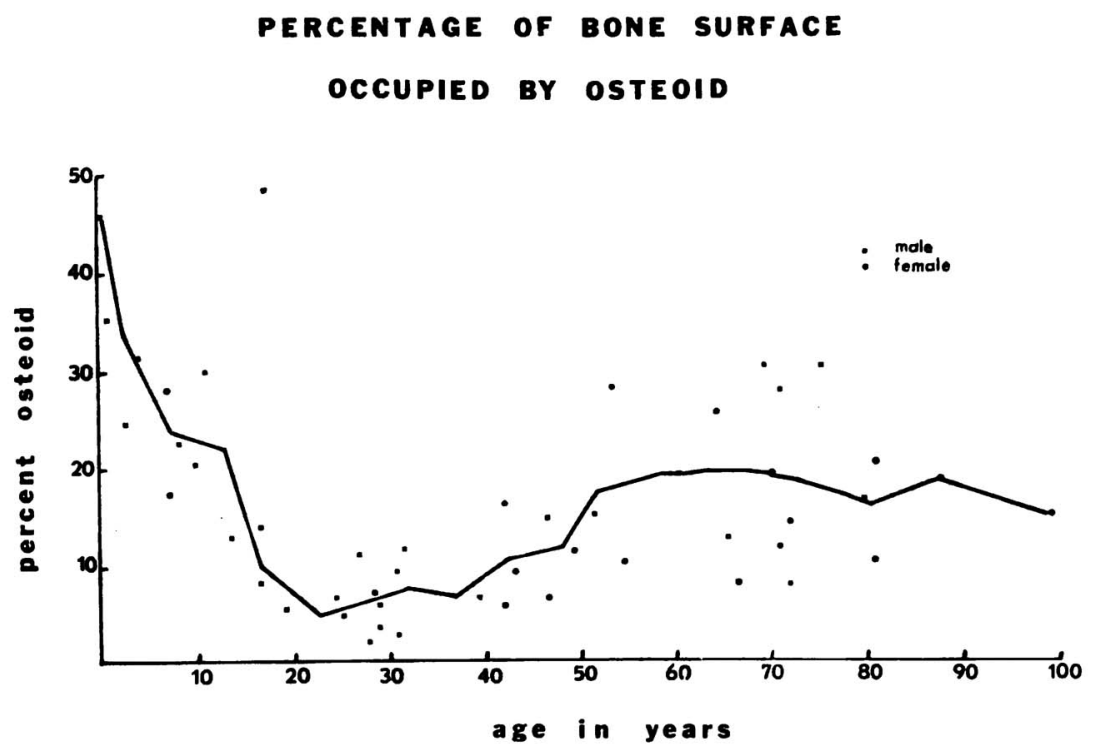

Fig.4. 
Platè II

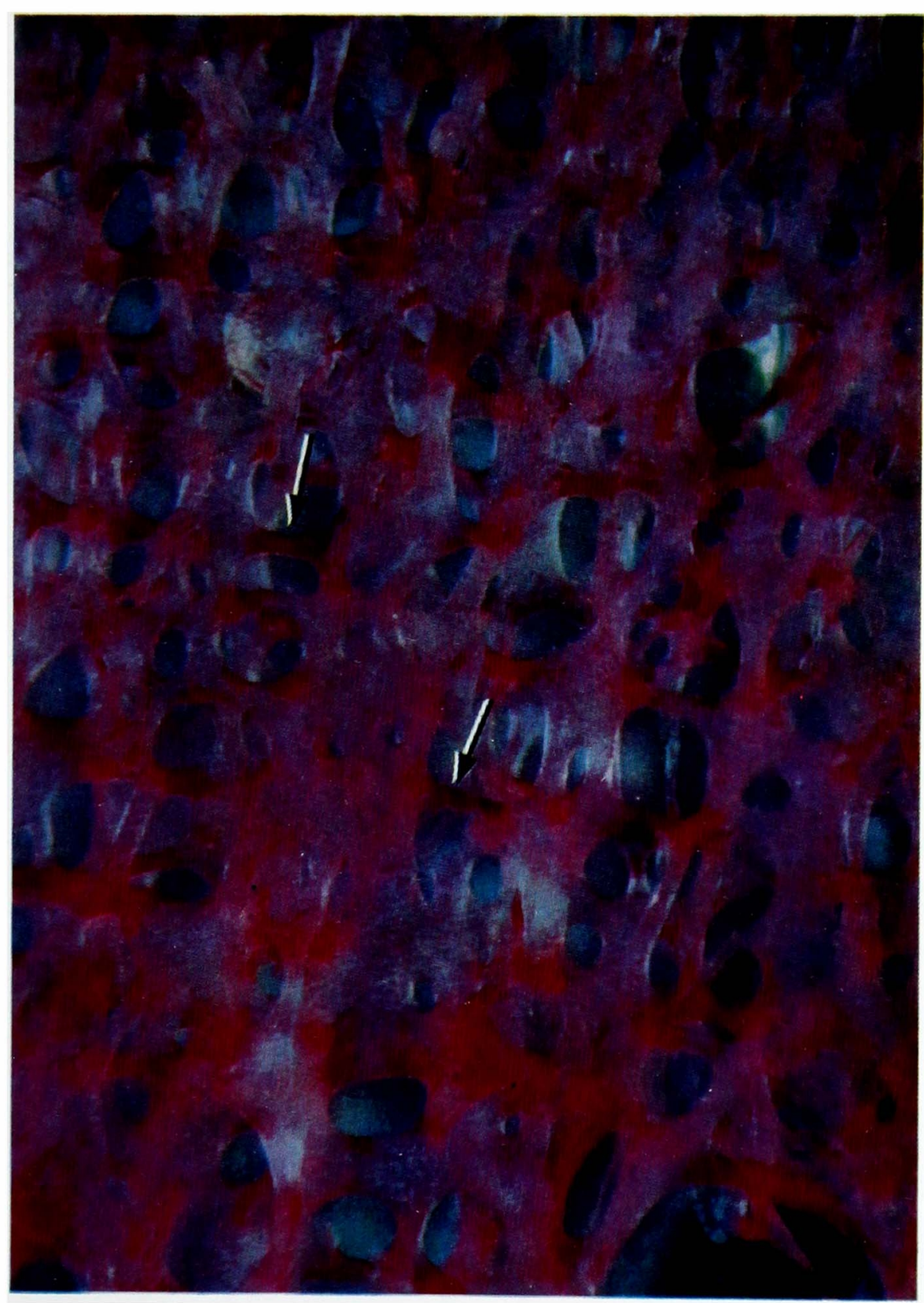

Fig.5.

J.-K. Wei and J.S. Arnold 
Plate IV

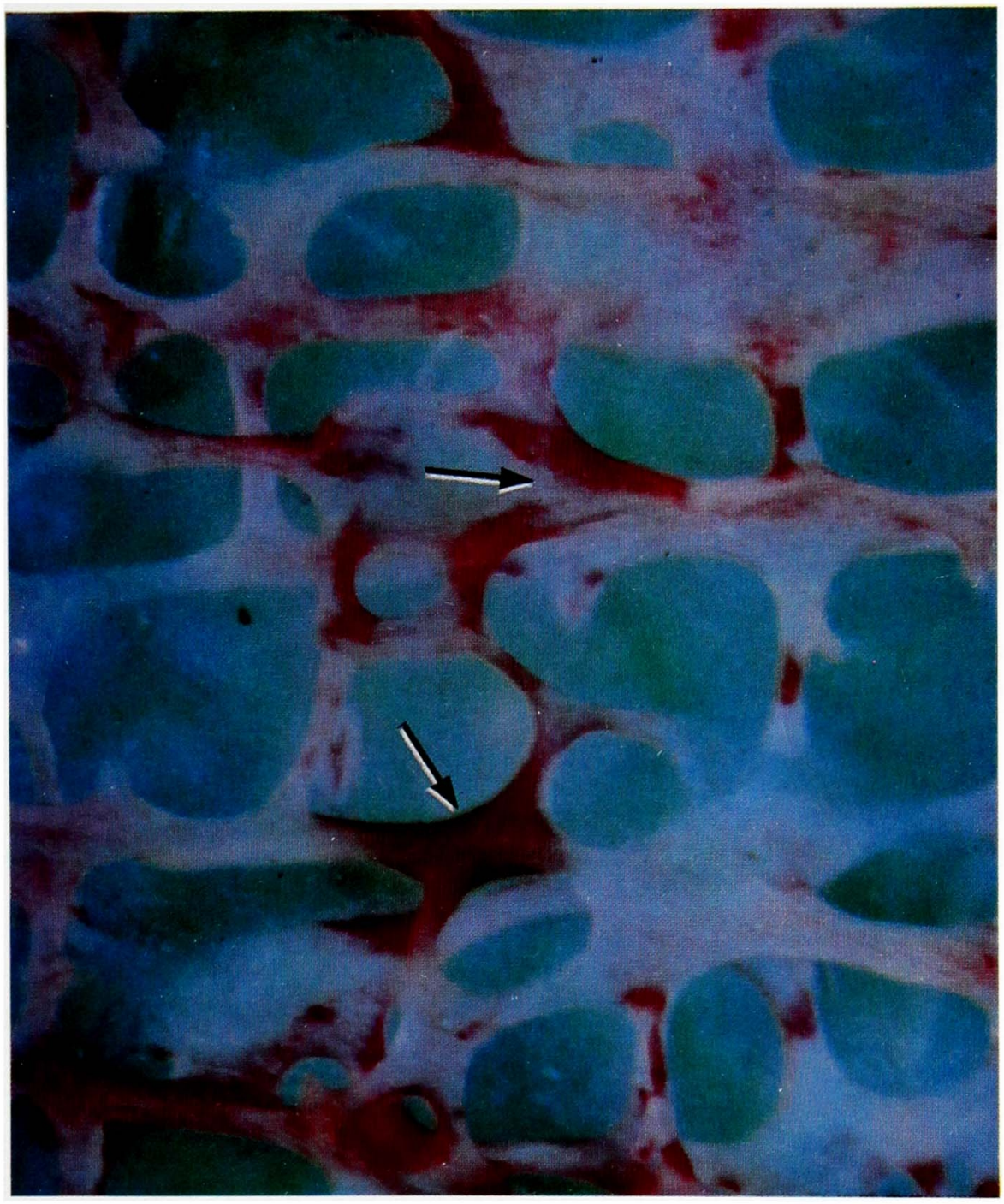

Fig.6. 